\title{
Closed-Form Bounds for Multihop Relayed Communications in Nakagami- $m$ Fading
}

\author{
George K. Karagiannidis*, Theodoros A. Tsiftsis ${ }^{\dagger}$, Ranjan K. Mallik ${ }^{\ddagger}$, \\ Nikos C. Sagias ${ }^{\S}$, and Stavros A. Kotsopoulos ${ }^{\dagger}$

\begin{abstract}
*Department of Electrical \& Computer Engineering, Aristotle University of Thessaloniki, 54124 Thessaloniki, Greece, Email: geokarag@auth.gr ${ }^{\dagger}$ Department of Electrical \& Computer Engineering, University of Patras, Rion, 26500 Patras, Greece, Email: \{tsiftsis;kotsop\}@ee.upatras.gr

${ }_{\ddagger}^{\ddagger}$ Department of Electrical Engineering, Indian Institute of Technology, Hauz Khas, 110016 New Delhi, India, Email: rkmallik@ee.iitd.ernet.in
\end{abstract}

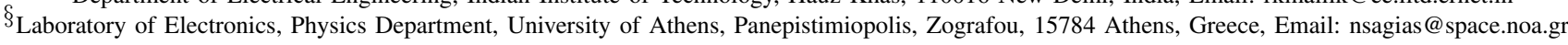

\begin{abstract}
We present closed-form bounds for the performance of multihop transmissions with non-regenerative relays over Nakagami- $m$ fading channels. The end-to-end signal-to-noise ratio (SNR) is formulated and upper bounded by using the inequality between harmonic and geometric mean of positive random variables (RVs). Novel closed-form expressions are derived for the moment generating function, the probability density function, and the cumulative distribution function of the product of arbitrary powers of statistically independent Gamma RVs in terms of the Meijer's G-function. Using these theoretical results, closed-form lower bounds are obtained for the outage and average bit error probability of phase or frequency modulated signallings, while simple asymptotic expressions are also given for the bounds at high SNRs. Numerical results are compared to computer simulations, to show the tightness of the proposed bounds.
\end{abstract}

\section{INTRODUCTION}

Multihop systems realize a number of advantages over traditional communications systems in the areas of deployment, connectivity and capacity while minimize the need for fixed infrastructure. Relaying techniques enable network connectivity where traditional architectures are impractical due to location constraints and can be applied to cellular, wireless local area networks (WLAN), and hybrid networks. In multihop systems the source-terminal communicates with the destination-terminal through a number of relays-terminals, having the advantage of broadening the coverage without using large transmitting power [1]-[5]. The concept of cooperative diversity, where the mobile users cooperate each other in order to exploit the benefits of spatial diversity without the need of using physical antenna arrays, has also gained great interest [6]-[9].

The performance analysis of multihop wireless communication systems operating in fading channels has been an important field of research in the past few years. Hasna and Alouini have presented a useful and semi-analytical framework for the evaluation of the end-to-end outage probability of multihop wireless systems with non-regenerative channel state information (CSI)-assisted relays over Nakagami- $m$ fading channels [3]. Moreover, the same authors have studied the dual-hop systems with regenerative and non-regenerative (CSIassisted or fixed gain) relays over Rayleigh [1], [4] and
Nakagami-m [2] fading channels. Recently, Boyer et al. [5], have proposed and characterized four channel models for multihop wireless communication and also have introduced the concept of multihop diversity. Finally, Karagiannidis et al. have studied the performance bounds for multihop wireless communications with blind (fixed gain) relays over Rice, Hoyt and Nakagami- $m$ fading channels [10], using the momentsbased approach [11]. However, to the best of the authors knowledge, the performance of multihop relayed systems has never been addressed in terms of tabulated functions in Nakagami- $m$ fading.

In this paper, using the well-known inequality between harmonic and geometric means of positive random variables (RVs), we present efficient performance bounds for the end-toend signal-to-noise ratio (SNR) of multihop wireless communication systems with CSI-assisted or fixed gain relays operating in non-identical Nakagami- $m$ fading channels. Motivated by the fact that the proposed bounds, in their general form, are products of arbitrary powers of statistically independent squared Nakagami-m (Gamma) RVs, we derive novel closedform expressions for their moment generating function (MGF), probability density function (PDF), and cumulative distribution function (CDF) in terms of the Meijer's G-function. Using these expressions, closed-form lower bounds are presented for important end-to-end system performance metrics, such as outage probability and average bit error probability (ABEP) for binary phase shift-keying (BPSK) and binary frequency shiftkeying (BFSK) modulation schemes, while simple asymptotic expressions are also given for the bounds at high SNRs. Numerical and computer simulation examples verify the accuracy of the presented mathematical analysis and show the tightness of the proposed bounds.

\section{STATISTICAL BACKGROUND}

Theorem 1: (MGF of the product of arbitrary powers of Gamma $R V s$ ): Let $\left\{X_{i}\right\}_{i=1}^{N}$ be $N$ independent, but not necessarily identically distributed (i.n.i.d.), Gamma RVs, with PDF given by

$$
f_{X_{i}}(x)=\frac{x^{\alpha_{i}-1}}{\beta_{i}^{\alpha_{i}} \Gamma\left(\alpha_{i}\right)} \exp \left(-\frac{x}{\beta_{i}}\right)
$$




$$
\mathcal{M}_{Y_{1}}(s)=\frac{\sqrt{k} \prod_{i=1}^{N} \ell_{i}^{\alpha_{i}-1 / 2}}{(\sqrt{2 \pi})^{r-N+k-1} \prod_{i=1}^{N} \Gamma\left(\alpha_{i}\right)} G_{r, k}^{k, r}\left[\frac{(-1)^{k}(s / k)^{k}}{\prod_{i=1}^{N}\left(\beta_{i} \ell_{i}\right)^{-\ell_{i}}} \mid \begin{array}{c}
\Delta\left(\ell_{1}, 1-\alpha_{1}\right), \Delta\left(\ell_{2}, 1-\alpha_{2}\right), \ldots, \Delta\left(\ell_{N}, 1-\alpha_{N}\right) \\
\Delta(k, 0)
\end{array}\right]
$$

where $\Gamma(\cdot)$ is the Gamma function [12, eq. (8.310/1)] and $\alpha_{i}, \beta_{i}>0$. Then, the MGF of the new RV $Y_{1}$, defined as the product of arbitrary powers of $N$ RVs $X_{i}$, i.e.,

$$
Y_{1} \triangleq \prod_{i=1}^{N} X_{i}^{\ell_{i} / k}
$$

with $\ell_{1}, \ell_{2}, \ldots, \ell_{N}$ and $k$, being positive integers, can be expressed in closed-form as in (3) (see at top of this page), where $r=\sum_{i=1}^{N} \ell_{i}, \Delta(k, u) \triangleq u / k,(u+1) / k, \ldots,(u+k-1) / k$, with $u$ a real constant, and $G[\cdot]$ is the Meijer's G-function [12, eq. (9.301)].

Note, that Meijer's G-function is a standard built-in function in most of the well-know mathematical software packages such as in MAPLE and MATHEMATICA. In addition, using [13, eq. (18)], it can be written in terms of the more familiar generalized hypergeometric functions [12, eq. (9.14/1)].

Proof: See in [14, Appendix].

Corollary 1: (PDF of the product of arbitrary powers of Gamma $R V s$ ): The PDF of $Y_{1}$ is given by

$$
\begin{aligned}
& f_{Y_{1}}(y)=\frac{k y^{-1} \prod_{i=1}^{N} \ell_{i}^{\alpha_{i}-1 / 2}}{(\sqrt{2 \pi})^{r-N} \prod_{i=1}^{N} \Gamma\left(\alpha_{i}\right)} \\
& \times G_{0, r}^{r, 0}\left[y^{k} \prod_{i=1}^{N}\left(\frac{1}{\beta_{i} \ell_{i}}\right)^{\ell_{i}} \mid \frac{-}{\Phi_{1}, \Phi_{2}, \ldots, \Phi_{N}}\right]
\end{aligned}
$$

where $\Phi_{i} \triangleq \Delta\left(\ell_{i}, \alpha_{i}\right)$.

Proof: The PDF of $Y_{1}$ can be derived as $f_{Y_{1}}(y)=$ $\mathcal{L}^{-1}\left\{M_{Y_{1}}(-s) ; y\right\}$ where $\mathcal{L}^{-1}(\cdot ; \cdot)$ denotes the inverse Laplace transform. Using the formula for the inverse Laplace transform of the Meijer's G-function [15, eq. (3.38.1)], we obtain (4).

It must be mentioned here, that $f_{Y_{1}}(\cdot)$ represents a valid PDF expression, since is a non-negative function and using [13, eq. (24)] and [16, eq. (6.1.20)], it can be easily verified that $\int_{0}^{\infty} f_{Y_{1}}(y) d y=1$.

In Fig. 1, we plot $f_{Y_{1}}(y)$, while Monte Carlo simulations have been performed and included in the same figure to show the correctness of the numerical evaluation. From this comparison, it is evident an excellent match between simulation and analytical results.

Lemma 1 (PDF of the product of Gamma RVs): The PDF of the product of $N$ i.n.i.d. Gamma RVs, $Y_{2} \triangleq \prod_{i=1}^{N} X_{i}$,

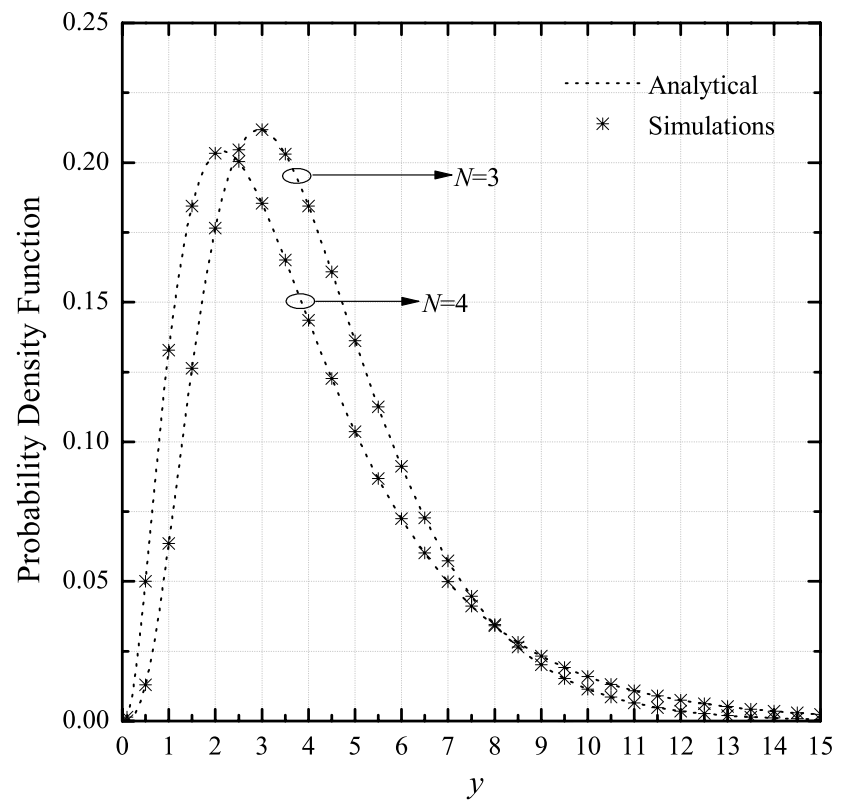

Fig. 1. Comparison between analytical and Monte Carlo simulations results, for the PDF formulated by (4) $\left(k=3, \ell_{i}=i, \beta_{1}=10, \beta_{2}=\beta_{3}=1.05\right.$, and $\beta_{4}=0.25$ ).

can be derived by setting $\ell_{i}=k=1$ in (4) as

$$
f_{Y_{2}}(y)=\frac{y^{-1}}{\prod_{i=1}^{N} \Gamma\left(\alpha_{i}\right)} G_{0, N}^{N, 0}\left[\frac{y}{\prod_{i=1}^{N} \beta_{i}} \mid \begin{array}{c}
- \\
\alpha_{1}, \alpha_{2}, \ldots, \alpha_{N}
\end{array}\right]
$$

For $N=2$, and using [13, eq. (18)], (5) is reduced to the formula presented by Shin and Lee [17, Appendix A, eq. (31)].

Corollary 2: (CDF of the product of arbitrary powers of Gamma RVs): The CDF of $Y_{1}$ is given by

$$
\begin{aligned}
& F_{Y_{1}}(y)=\frac{\prod_{i=1}^{N} \ell_{i}^{\alpha_{i}-1 / 2}}{(\sqrt{2 \pi})^{r-N} \prod_{i=1}^{N} \Gamma\left(\alpha_{i}\right)} \\
& \times G_{1, r+1}^{r, 1}\left[y^{k} \prod_{i=1}^{N}\left(\frac{1}{\beta_{i} \ell_{i}}\right)^{\ell_{i}} \mid \begin{array}{c}
1 \\
\left.\Phi_{1}, \Phi_{2}, \ldots, \Phi_{N}, 0\right] .
\end{array}\right.
\end{aligned}
$$

Proof: With the aid of $F_{Y_{1}}(y)=\int_{0}^{y} f_{Y_{1}}(z) d z$ and using [13, eq. (26)], yields (6).

Lemma 2 (CDF of the product of Gamma RVs): The CDF 
of $Y_{2}$ can be derived by setting $\ell_{i}=k=1$ in (6), resulting in

$$
F_{Y_{2}}(y)=\frac{G_{1, N+1}^{N, 1}\left[y / \prod_{i=1}^{N} \beta_{i} \mid \begin{array}{c}
1 \\
\alpha_{1}, \alpha_{2}, \ldots, \alpha_{N}, 0
\end{array}\right]}{\prod_{i=1}^{N} \Gamma\left(\alpha_{i}\right)} .
$$

\section{UPPER BOUNDS FOR THE END-TO-END SNRS}

In this section, we derive upper bounds for the distributions of the end-to-end SNR for the CSI-assisted and fixed gain relay implementations of a multihop communication system.

\section{A. System and Channel Model}

We consider an $N$-hop wireless communication system which operates over i.n.i.d. Nakagami- $m$ fading channels. The source terminal $\mathrm{S}$ communicates with the destination terminal D through $N-1$ nodes-terminals, $R_{1}, R_{2}, \ldots, R_{N-1}$. These terminals act as intermediate non-regenerative relays from one hop to the next. It is also assumed that all nodesrelays can simultaneously receive and transmit (in the same frequency band), and no delay is incurred in the whole chain of transmissions. Assume that terminal $\mathrm{S}$ is transmitting a signal with an average power normalized to unity. Then, the end-toend SNR, i.e., the SNR at D, can be written as [3]

$$
\gamma_{\text {end }}=\frac{\prod_{i=1}^{N} v_{i}^{2} g_{i-1}^{2}}{\sum_{i=1}^{N} N_{0, i}\left(\prod_{j=i+1}^{N} g_{j-1}^{2} v_{j}^{2}\right)}
$$

where $v_{i}$ is the fading amplitude of the $i$ th hop, $N_{0, i}$ is the one sided power spectral density at the input of the $i$ th relay, and $g_{i}$ is the gain of the $i$ th relay with $g_{0}=1$.

Due to the fact that, $v_{i}$ is Nakagami- $m$ distributed, the corresponding instantaneous SNR, $\gamma_{i}$, defined as $\gamma_{i}=v_{i}^{2} / N_{0, i}$, is Gamma distributed, with PDF given by [18]

$$
f_{\gamma_{i}}(\gamma)=\frac{m_{i}^{m_{i}}}{\bar{\gamma}_{i}^{m_{i}} \Gamma\left(m_{i}\right)} \gamma^{m_{i}-1} \exp \left(-m_{i} \frac{\gamma}{\bar{\gamma}_{i}}\right)
$$

where $m_{i} \geq 1 / 2$ is a parameter describing the fading severity of the $i$ th hop and $\bar{\gamma}_{i}$ is the average SNR $\bar{\gamma}_{i}=E\left\langle v_{i}^{2}\right\rangle / N_{0, i}$, with $E\langle\cdot\rangle$ denoting expectation. It is obvious, that by setting $\alpha_{i}=m_{i}$ and $\beta_{i}=\bar{\gamma}_{i} / m_{i}$ in (1), yields (9).

\section{B. CSI-Assisted Relays}

One choice of gain is proposed in [1]-[3] as

$$
g_{i}^{2}=\frac{1}{v_{i}^{2}}
$$

where the relay just amplifies the incoming signal with the inverse of the channel of the previous hop regardless the fading state (i.e., the noise) of that hop. As mentioned in [1]-[3], such a kind of relay serves as benchmark for all practical multihop systems using non-regenerative relays and its performance, in the high SNR region, is equal to the performance of the CSIassisted relays which satisfy the average power constraint, with an amplifying gain given by [9, eq. (9)]

$$
g_{i}^{2}=\frac{1}{v_{i}^{2}+N_{0, i}} \text {. }
$$

By applying (10) to (8), the end-to-end SNR becomes

$$
\gamma_{\text {end }}=\left(\sum_{i=1}^{N} \frac{1}{\gamma_{i}}\right)^{-1}
$$

In order to study important performance metrics of the endto-end SNR, (12) should be expressed in a more mathematically tractable form. To achieve it, we propose an upper bound for (12) using the well-known inequality between geometric and harmonic mean of $N$ positive RVs $x_{1}, x_{2}, \ldots, x_{N}$ given by

$$
\mathcal{H}_{N} \leq \mathcal{G}_{N}
$$

where $\mathcal{H}_{N} \triangleq N\left(\sum_{i=1}^{N} 1 / x_{i}\right)^{-1}$ and $\mathcal{G}_{N} \triangleq \prod_{i=1}^{N} x_{i}^{1 / N}$ are the harmonic and geometric means, respectively. In (13), the equality holds only when $x_{1}=x_{2}=\cdots=x_{N}$. Using (12) and (13), an upper bound for the end-to-end SNR, $\gamma_{b}$, for multihop systems with CSI-assisted relays can be obtained as

$$
\gamma_{e n d} \leq \gamma_{b}=\frac{1}{N} \prod_{i=1}^{N} \gamma_{i}^{1 / N}
$$

Applying (4) and (6) in (14), the PDF and CDF of $\gamma_{b}$ can be written in closed-form as

$$
f_{\gamma_{b}}(\gamma)=\frac{N G_{0, N}^{N, 0}\left[\gamma^{N} N^{N} \prod_{i=1}^{N} \frac{m_{i}}{\bar{\gamma}_{i}} \mid m_{1}, m_{2}, \ldots, m_{N}\right]}{\gamma \prod_{i=1}^{N} \Gamma\left(m_{i}\right)}
$$

and

$$
F_{\gamma_{b}}(\gamma)=\frac{G_{1, N+1}^{N, 1}\left[\gamma^{N} N^{N} \prod_{i=1}^{N} \frac{m_{i}}{\bar{\gamma}_{i}} \mid m_{1}, m_{2}, \ldots, m_{N}, 0\right]}{\prod_{i=1}^{N} \Gamma\left(m_{i}\right)}
$$

respectively.

\section{Fixed Gain Relays}

The fixed gain relays provide reduced implementation complexity in the CSI part, in expense of the requirements for high transmission power amplifiers which may be very expensive in practice. Non-regenerative relays introduce fixed gains to the received signal given by

$$
g_{i}^{2}=\frac{1}{C_{i} N_{0, i}}
$$

where $C_{i}$ is positive a constant $\left(C_{0}=1\right)$. Following the same procedure as in [3] and using (17), the end-to-end SNR can 
be expressed as [10]

$$
\gamma_{e n d}^{\prime}=\left(\sum_{n=1}^{N} \prod_{j=1}^{n} \frac{C_{j-1}}{\gamma_{j}}\right)^{-1} .
$$

Using (13), an upper bound for the end-to-end SNR when fixed gain relays are used, can be written as

$$
\gamma_{\text {end }}^{\prime} \leq \gamma_{b}^{\prime}=\mathcal{Z}_{N} \prod_{i=1}^{N} \gamma_{i}^{\frac{N+1-i}{N}}
$$

where $\mathcal{Z}_{N}$ is a constant related to the introduced fixed gain and given by

$$
\mathcal{Z}_{N}=\frac{1}{N} \prod_{i=1}^{N} C_{i}^{-\frac{N-i}{N}}
$$

Using (4), (6), and (19), and after much laborious manipulations, the PDF and CDF of $\gamma_{b}^{\prime}$ can be obtained, respectively, as

$$
f_{\gamma_{b}^{\prime}}(\gamma)=\frac{N \mathcal{P}}{\gamma} G_{0, \varrho}^{\varrho, 0}\left[\begin{array}{l|c}
\mathcal{R} \gamma^{N} & - \\
\Lambda_{1}, \Lambda_{2}, \ldots, \Lambda_{N}
\end{array}\right]
$$

and

$$
F_{\gamma_{b}^{\prime}}(\gamma)=\mathcal{P} G_{1, \varrho+1}^{\varrho, 1}\left[\mathcal{R} \gamma^{N} \mid \begin{array}{c}
1 \\
\Lambda_{1}, \Lambda_{2}, \ldots, \Lambda_{N}, 0
\end{array}\right]
$$

where $\varrho=N(N+1) / 2, \Lambda_{i}=\Delta\left(N+1-i, m_{i}\right)$,

$$
\mathcal{P}=\frac{\prod_{i=1}^{N}(N+1-i)^{m_{i}-1 / 2}}{(\sqrt{2 \pi})^{N(N-1) / 2} \prod_{i=1}^{N} \Gamma\left(m_{i}\right)}
$$

and

$$
\mathcal{R}=\mathcal{Z}_{N}^{-N} \prod_{i=1}^{N}\left[\frac{m_{i}}{\bar{\gamma}_{i}(N+1-i)}\right]^{N+1-i} .
$$

\section{Performance Metrics}

Using the formulae proposed in the previous section, we present bounds for the outage probability and the ABEP of BPSK and BFSK signallings for both CSI-assisted and fixed gain relays.

\section{A. Outage Probability}

The probability of outage is defined as the probability that the instantaneous SNR falls below a specified threshold $\gamma_{t h}$. This threshold is a protection value of the SNR above which the quality of service is satisfactory. In case of the multihop systems under consideration, the use of upper bounds $\gamma_{b}$ or $\gamma_{b}^{\prime}$ leads to lower bounds for the outage probability in the destination terminal D, expressed as $P_{\text {out }} \geq F_{\gamma_{b}}\left(\gamma_{t h}\right)$ for CSIassisted relays and $P_{\text {out }}^{\prime} \geq F_{\gamma_{b}^{\prime}}\left(\gamma_{t h}\right)$ for fixed gain relays.

As an indicative example for the proposed bounds, assuming equal average SNRs per hop for all hops $\bar{\gamma}_{i}=\bar{\gamma}$, in Fig. 2, lower bounds for the outage probability, when fixed gain relays are assumed, are plotted as a function of the inverse normalized to outage threshold, $\bar{\gamma} / \gamma_{t h}$. The obtained results clearly show that the outage performance degrades with an

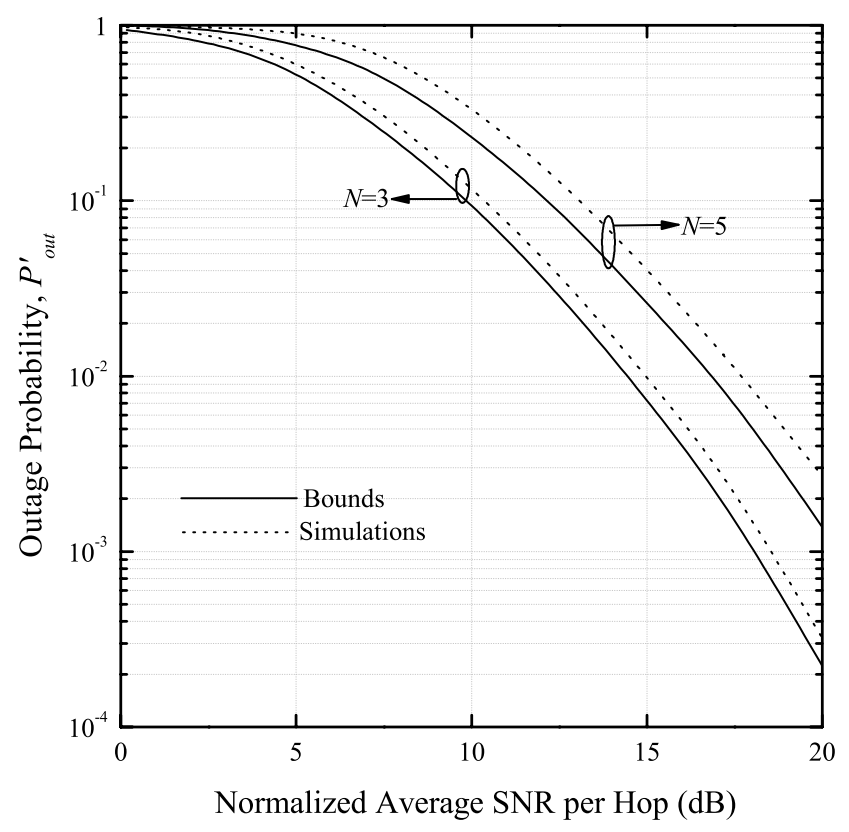

Fig. 2. Outage probability bounds for a multihop system with fixed gain relays $\left(\bar{\gamma}_{i}=\bar{\gamma}, C_{i}=1.7\right.$, and $\left.m_{i}=m=2.7\right)$.

increase of the number of hops. Additionally, the lower the value of $N$, the tighter the proposed bounds are, even for high SNR values.

\section{B. Average Bit Error Probability}

For coherent binary signal constellations, the ABEP, $P_{e}$, can be formulated as [18]

$$
P_{e}=\frac{1}{2} E\langle\operatorname{erfc}(\sqrt{\xi \gamma})\rangle
$$

where $\operatorname{erfc}(\cdot)$ is the complementary error function [12, eq. (8250/4)], $\xi=(1-\varepsilon) / 2$ where $\varepsilon$ being the correlation coefficient between the two signaling waveforms. Thus, for $\varepsilon=-1, \xi=1$ for coherent BPSK and for $\varepsilon=0, \xi=1 / 2$ for coherent orthogonal BFSK.

1) CSI-assisted relays: Using (15), (23), the Meijer's G-function representation of the $\operatorname{erfc}(\cdot)$ function $[19$, eq. (06.27.26.0006.01)], and [13, eq. (21)], a lower bound for ABEP of CSI-assisted relays over Nakagami- $m$ fading channels can be expressed in closed-from as

$$
\begin{aligned}
& P_{e, \gamma_{b}}=\frac{(2 \pi)^{-N / 2}}{\sqrt{2} \prod_{i=1}^{N} \Gamma\left(m_{i}\right)} \times \\
& G_{2 N, 2 N}^{N, 2 N}\left[\left(\frac{N^{2}}{\xi}\right)^{N} \prod_{i=1}^{N} \frac{m_{i}}{\bar{\gamma}_{i}} \mid \begin{array}{c}
\Delta(N, 1), \Delta(N, 1 / 2) \\
m_{1}, m_{2}, \ldots, m_{N}, \Delta(N, 0)
\end{array}\right] .
\end{aligned}
$$

In Fig. 3, lower bounds for the ABEP of a multihop system with CSI-assisted relays are plotted versus the average SNR per hop $\bar{\gamma}$. Again here, it is evident that the proposed bounds are accurate and tight and as expected, the ABEP deteriorates with an increase in the number of hops. 


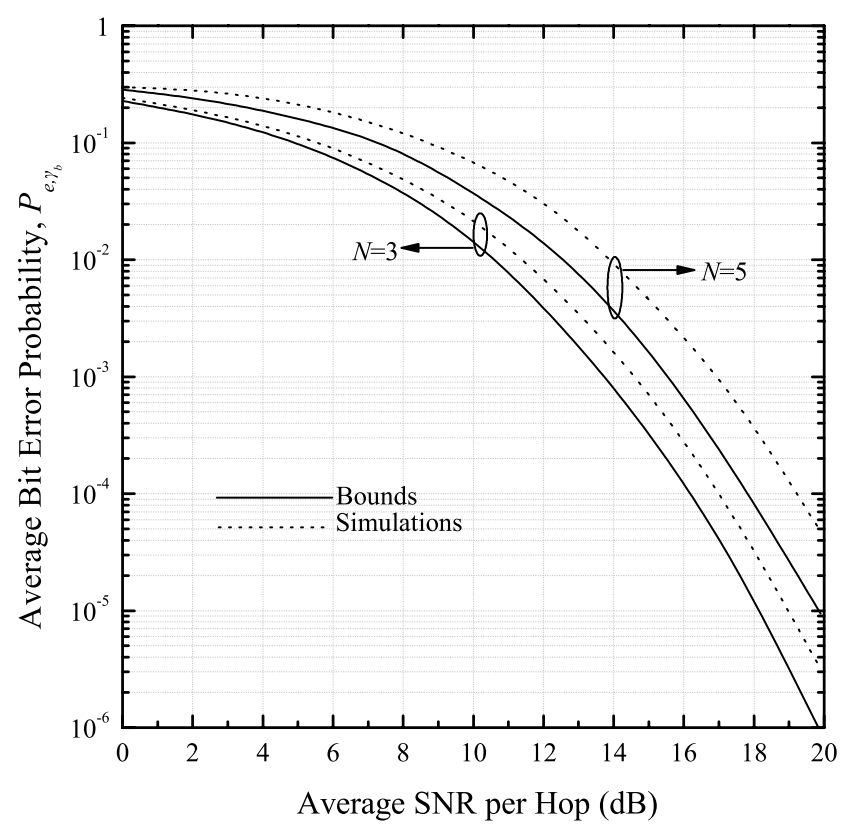

Fig. 3. BPSK error bounds for a multihop system with CSI-assisted relays in Nakagami- $m$ fading $\left(\bar{\gamma}_{i}=\bar{\gamma}\right.$ and $\left.m_{i}=m=2.7\right)$.

2) Fixed gain relays: For the case of fixed gain relays, a lower bound for ABEP can be found using (21) and (23) as

$$
\begin{aligned}
P_{e, \gamma_{b}^{\prime}}= & \frac{\sqrt{\pi} \mathcal{P}}{(\sqrt{2 \pi})^{(N+1)}} \\
& \times G_{2 N, \varrho+N}^{\varrho, 2 N}\left[\begin{array}{l|l}
\frac{\mathcal{R}}{N^{-N}} & \Delta(N, 1), \Delta(N, 1 / 2) \\
\Lambda_{1}, \ldots, \Lambda_{N}, \Delta(N, 0)
\end{array}\right] .
\end{aligned}
$$

\section{Asymptotic Bounds For High Average SNRs per Hop}

For high average SNRs per hop, the arguments of the Meijer's G-function in outage probability and ABEP expressions tend to zero. Hence, following an asymptotic expansion of the Meijer's G-function [19, eq. (07.34.06.0006.01)]

$$
\begin{aligned}
& G_{p, q}^{m, n}\left[z \mid \begin{array}{l}
a_{1}, \ldots, a_{n}, a_{n+1}, \ldots, a_{p} \\
b_{1}, \ldots, b_{m}, b_{m+1}, \ldots, b_{q}
\end{array}\right]= \\
& \sum_{k=1}^{m} \frac{\prod_{j=1, j \neq k}^{m} \Gamma\left(b_{j}-b_{k}\right) \prod_{j=1}^{n} \Gamma\left(1-a_{j}+b_{k}\right)}{\prod_{j=n+1}^{p} \Gamma\left(a_{j}-b_{k}\right) \prod_{j=m+1}^{q} \Gamma\left(1-b_{j}+b_{k}\right)} z^{b_{k}}
\end{aligned}
$$

where $a_{i}, b_{i}$, and $z>0$ are arbitrary real values and $m, n$, $p$, and $q$ are arbitrary positive integers, any of the derived performance metrics may be used in conjunction with (26) to derive corresponding simple closed-form expressions for any known type of CSI-assisted or fixed gain relays, operating in the high SNR region.

\section{Conclusions}

The performance of multihop systems with non-regenerative relays operating over Nakagami- $m$ fading channels was stu- died. The end-to-end SNR was upper bounded by using the inequality between harmonic and geometric mean and tight lower bounds for the outage probability and ABEP of BPSK and BFSK signallings were obtained in closed-forms. From these results, it was concluded that the proposed bounds were very tight, especially for low values of $N$ which have practical interest. Several numerical examples were also presented and compared to corresponding exact computer simulations results, to show the tightness of the proposed bounds.

\section{REFERENCES}

[1] M. O. Hasna and M. S. Alouini, "End-to-end performance of transmission systems with relays over Rayleigh fading channels," IEEE Trans. Wireless Commun., vol. 2, pp. 1126-1131, Nov. 2003.

[2] - "Harmonic mean and end-to-end performance of transmission systems with relays," IEEE Trans. Commun., vol. 52, pp. 130-135, Jan. 2004.

[3] — "Outage probability of multihop transmission over Nakagami fading channels," IEEE Commun. Lett., vol. 7, pp. 216-218, May 2003.

[4] _ - "A performance study of dual-hop transmissions with fixed gain relays," IEEE Trans. Wireless Commun., vol. 3, pp. 1963-1968, Nov. 2004.

[5] J. Boyer, D. D. Falconer, and H. Yanikomeroglu, "Multihop diversity in wireless relaying channels," IEEE Trans. Commun., vol. 52, pp. 1820 1830, Oct. 2004

[6] A. Sendonaris, E. Erkrip, and B. Aazhang, "User cooperation diversityPart I: System description,” IEEE Trans. Commun., vol. 51, pp. $1927-$ 1938, Nov. 2003.

[7] — , "User cooperation diversity-Part II: Implementation aspects and performance analysis," IEEE Trans. Commun., vol. 51, pp. 1927-1938, Nov. 2003.

[8] P. A. Anghel and M. Kaveh, "Exact symbol error probability of a cooperative network in a Rayleigh-fading environment," IEEE Trans. Wireless Commun., vol. 3, pp. 1416-1421, Sept. 2004.

[9] J. N. Laneman, D. N. C. Tse, and G. W. Wornell, "Cooperative diversity in wireless networks efficient protocols and outage behaviour," IEEE Trans. Inform. Theory, vol. 50, pp. 3062-3080, Dec. 2004.

[10] G. K. Karagiannidis, D. A. Zogas, N. C. Sagias, T. A. Tsiftsis, and P. T. Mathiopoulos, "Multihop communications with fixed-gain relays over generalized fading channels," in Proc. IEEE Global Telecommunications Conference, vol. 1, Dallas, Texas, USA, Dec. 2004, pp. 36-40.

[11] G. K. Karagiannidis, "Moments-based approach to the performance analysis of equal-gain diversity in Nakagami- $m$ fading," IEEE Trans. Commun., vol. 52, pp. 685-690, May 2004.

[12] I. S. Gradshteyn and I. M. Ryzhik, Table of Integrals, Series, and Products, 6th ed. New York: Academic, 2000.

[13] V. S. Adamchik and O. I. Marichev, "The algorithm for calculating integrals of hypergeometric type functions and its realization in REDUCE system," in Proc. International Conference on Symbolic and Algebraic Computation, Tokyo, Japan, 1990, pp. 212-224.

[14] G. K. Karagiannidis, T. A. Tsiftsis, and R. K. Mallik, "Bounds of multihop relayed communications in Nakagami- $m$ fading," IEEE Trans. Commun., submitted for publication.

[15] A. P. Prudnikov, Y. A. Brychkov, and O. I. Marichev, Integral and Series. Vol. 5: Inverse Laplace Transforms, 5th ed. London, UK: Gordon and Breach, 1992.

[16] M. Abramovitz and I. A. Stegun, Handbook of Mathematical Functions with Formulas, Graphs, and Mathematical Tables, 9th ed. New York: Dover, 1972.

[17] H. Shin and J. H. Lee, "Performance analysis of space time block codes overkeyhole Nakagami- $m$ fading channels," IEEE Trans. Veh. Technol., vol. 53, pp. 416-424, Mar. 2004.

[18] M. K. Simon and M.-S. Alouini, Digital Communication over Fading Channels, 2nd ed. New York: Wiley, 2004.

[19] Wolfram. (2004) The Wolfram functions site. Internet. [Online]. Available: http://functions.wolfram.com/ 